\title{
Atlantoaxial instability in Down's syndrome: a five year follow up study
}

\author{
R E Morton, M Ali Khan, C Murray-Leslie, S Elliott
}

\begin{abstract}
In 1986 all 90 children aged 4-19 years with Down's syndrome attending school in the area served by the Southern Derbyshire Health Authority underwent radiography to identify atlantoaxial instability (AAI). This study details repeat observations five years later. Full results were available on $67(74 \%)$, information on health status was available on the remaining $19(21 \%)$; four (4\%) were untraced.

There was an overall significant reduction in the atlanto-axial gap over five years. No one developed AAI on repeat testing who had not had it earlier. One child who had previously had normal neck radiography developed acute symptomatic AAI after ear, nose, and throat surgery.

Radiographs were done on three occasions on the same day in 49 individuals, ensuring full flexion of the upper neck. There were no significant differences between the radiographs, even in five subjects with AAI.

Management of AAI in Down's syndrome is discussed in the light of these findings. Radiography can reliably detect children with chronic AAI who may be at risk of gradually developing symptoms; this may justify a screening programme. This must be distinguished from those who develop symptoms after acute trauma or anaesthesia, for which specific precautions are needed, and previous screening radiographs are unhelpful.

(Arch Dis Child 1995; 72: 115-119)
\end{abstract}

Childe MacKeith Centre and Derbyshire Children's Hospital R E Morton

Community Health Services, Southern Derbyshire

M Ali Khan

Department of Rehabilitation, Derbyshire Royal Infirmary

C Murray-Leslie

Department of Radiology, Derbyshire Children's Hospital and Derbyshire Royal Infirmary

$S$ Elliott

Correspondence to:

Dr Richard Morton, Ronnie MacKeith Child

Development Centre,

Derbyshire Royal Infirmary, London Road, Derby DE1 2QY.

Accepted 27 September 1994
Keywords: atlantoaxial instability, atlanto-axial gap, Down's syndrome.

In the past decade, there has been considerable concern about the high incidence of atlantoaxial instability (AAI) in Down's syndrome. However, while $10-20 \%$ of children with Down's syndrome have AAI radiologically (defined as an atlanto-axial gap of $4.0 \mathrm{~mm}$ or more), ${ }^{1}$ only around $18 \%$ of them have symptoms, ${ }^{2}$ including torticollis and varying signs of spinal cord compression. Symptoms develop gradually over the course of months or years, or acutely after specific trauma. The latter can occur in individuals with previously normal neck radiographs ${ }^{2}$ and this has lead to concern over their value. This confusion has been increased by doubts over the reliability of the radiographs themselves. Selby et al showed that when they were repeated on the same day in 19 subjects, six were abnormal on the first radiograph, and only three on the second. ${ }^{3}$ Clear distinction should be made between chronic and acute symptomatic AAI when considering the value of screening radiographs.

Long term studies are necessary to determine the natural history of AAI in Down's syndrome and how this relates to radiological measurements. In 1986 we screened all children living in our health authority with Down's syndrome aged 4-19 years for AAI, together with clinical assessment of signs and symptoms. ${ }^{4}$

In the present study we reviewed the radiographs and clinical evaluations on the same cohort of children five years later. All radiographs were taken with the neck fully flexed to maximise the atlanto-axial gap. If the neck is only flexed in the lower cervical region, flexion of the atlantoaxial joints is spared, and the gap may appear normal; variations in the way the neck is flexed for radiography may therefore account for discrepancies between measurements on radiographs. We assessed this in a subgroup by doing three radiographs 10 minutes apart, each with full flexion of the upper neck, and compared the measurements obtained.

\section{Subjects and methods}

To assess the reliability of neck radiographs in measuring the atlanto-axial gap, three different radiographs were taken sequentially in 49 children, including five with AAI. One was taken in the erect position and two supine, all 10 minutes apart. Maximal flexion of the upper neck was ensured on each occasion and to assist in this, a $45^{\circ}$ padded headrest was used for the supine studies (fig 1). The three radiographs were compared to find the maximal difference in the atlanto-axial gap between individual measurements. Results for these children were incorporated in the main study.

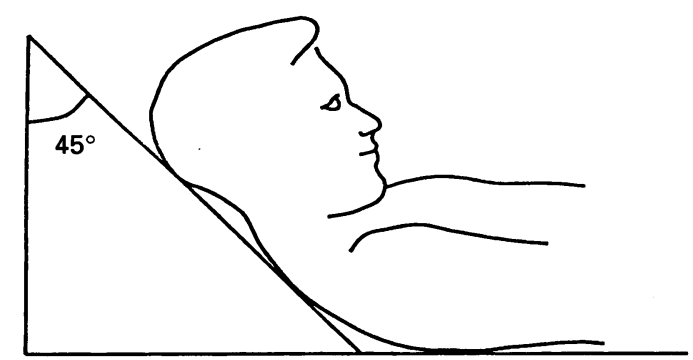

Figure 1 Use of headrest for supine neck radiograph showing position for maximal flexion of upper neck with neck back against headrest and chin tucked into chest. 


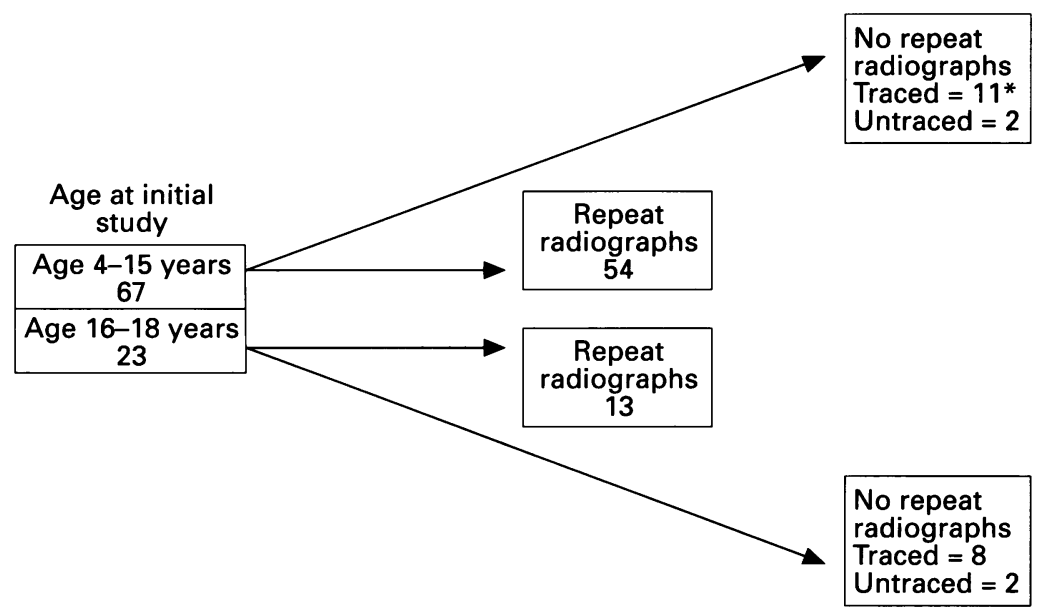

Figure 2 Follow up of 90 subjects with Down's syndrome after five years. ${ }^{\star}$ Includes one child with acute $A A I$, the only child to develop neck problems over the five year period.

In 1986, all individuals in school between the ages of 4 and 18 years with Down's syndrome, living in the Southern Derbyshire Health Authority, were examined for complications of AAI $(n=90)$, and had neck radiographs taken in the neutral, flexed, and erect positions. Of the 90 individuals, seven had significant AAI (gap over $4.0 \mathrm{~mm}$ ), but none had abnormal signs. In every case the atlantoaxial gap was greatest in the flexed position. Another two had abnormalities of the odontoid peg and 15 had odontoid hypoplasia. ${ }^{4}$ Those with significant AAI were advised not to participate in activities that would place undue stress on the neck.

Five years later these 90 individuals were traced and given an appointment for further clinical examination and neck radiography. If individuals did not attend, their general practitioner was asked to check the records for any evidence of symptomatic AAI. The examinations we performed consisted of checking for gait disturbance, neck movements, tendon reflexes, and plantar responses. One radiograph was taken in flexion, with the patient standing erect and a helper making sure the head was back and chin tucked into the chest, to ensure maximal flexion of the upper neck. The atlanto-axial gap was taken as the distance between the posterio-inferior aspect of the anterior arch of the atlas and the nearest adjacent surface of the odontoid process; measurements over $4.0 \mathrm{~mm}$ were taken as abnormal. The height of the odontoid was assessed using the method of McManners, measuring from the tip of the odontoid process to the superior aspect of the articular facets of the axis. ${ }^{5}$ Odontoid hypoplasia was considered to be

Outcome for seven individuals with significant $A A I$ (gap $>4.0 \mathrm{~mm}$ )

\begin{tabular}{lll}
\hline \multirow{2}{*}{$\begin{array}{l}\text { Initial age } \\
(\text { years })\end{array}$} & \multicolumn{2}{l}{ Atlanto-axial gap $(\mathrm{mm})$} \\
\cline { 2 - 3 } & Initial & After 5 years \\
\hline 6 & $5 \cdot 0$ & $3 \cdot 0$ \\
7 & $6 \cdot 0$ & $5 \cdot 0$ \\
8 & $5 \cdot 5$ & $5 \cdot 0$ \\
10 & $6 \cdot 0$ & $6 \cdot 0$ \\
14 & $6 \cdot 0$ & $5 \cdot 0$ \\
15 & $6 \cdot 0$ & $5 \cdot 0$ \\
\hline
\end{tabular}

present when the height of the odontoid was less than $2 \mathrm{SD}$ from the mean for normal children of the same age. ${ }^{6}$ Differences in the mean atlanto-axial gap in this population, over the five year period, were evaluated by Student's $t$ test.

This study was passed by our local ethics committee.

\section{Results}

Forty nine children had three radiographs (one erect and two supine), performed 10 minutes apart. In $31(63 \%)$, there was no difference in the measurement for the atlanto-axial gap. In the other $18(37 \%)$, there was a maximum $1 \cdot 0$ $\mathrm{mm}$ difference between two of the three films. Of these, the gap was greater on the erect radiograph alone in seven, one supine film alone in nine, and an erect and supine film in two.

From the initial cohort of 90 individuals, 67 were aged 4-15 and still at school at the time of the second study. The remaining 23 were 19 years or older for the second study and had left school. Details of the results of the follow up studies are given in fig 2. In total, 67 (74\%) had full repeat evaluation, including neck radiography. Nineteen $(21 \%)$ did not come for radiography, but a medical report was available from a general practitioner. The remaining four $(4 \%)$ had left the area.

Only one individual in the series showed symptoms of AAI over the five year period. This was a 9 year old boy who, after ear, nose, and throat surgery developed acute torticollis without neurological signs. Neck radiography indicated a rotatary dislocation at the atlantoaxial joint, with a gap of $7.0 \mathrm{~mm}$. Radiograph screening during the first study two years before this showed him to have a gap of only $3.0 \mathrm{~mm}$. He underwent surgical posterior fusion of the upper cervical vertebrae and made a full recovery.

Out of seven with AAI on radiography in 1986, five still had this in 1991, and they remained symptom free. No-one developed AAI in the latter study (that is, increased the gap to over $4.0 \mathrm{~mm}$ ), who had not had this five years before (table).

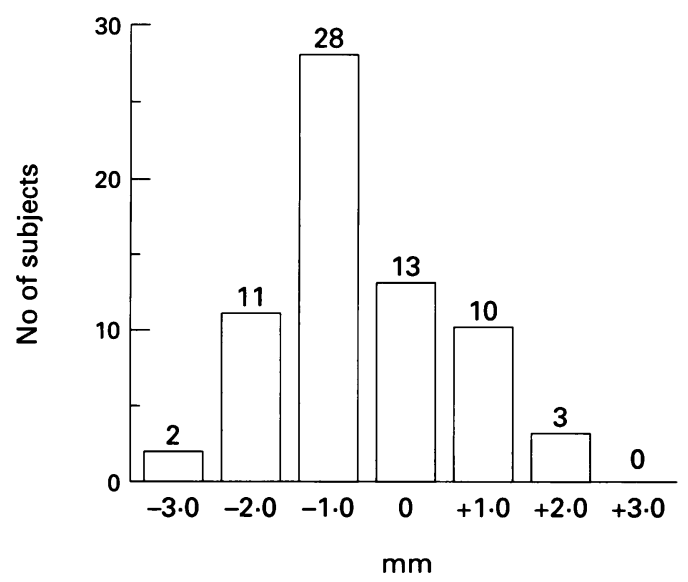

Figure 3 Changes in atlanto-axial gap over five years in 67 subjects. 
Sixty seven individuals received two radiographs five years apart and there was an overall significant reduction of the mean atlanto-axial gap from $2.95 \mathrm{~mm}$ (SD 1.18) to $2.45 \mathrm{~mm}$ (SD $1 \cdot 14)(p<0 \cdot 01)$. This trend is indicated in fig 3. Overall, 13 individuals showed an increase in the atlanto-axial gap over the five year period to a maximum of $2.0 \mathrm{~mm}$. Odontoid hypoplasia was found originally in 15 and was still present in three. Accessory ossicles were still present in the two children who had them in 1986.

\section{Discussion}

Our results indicate that radiographs can reliably measure the atlanto-axial gap if they are taken during full flexion of the upper neck. Variation between three radiographs taken on the same day showed a maximum difference of $1.0 \mathrm{~mm}$, even in the five individuals with AAI. Failure to ensure adequate upper neck flexion will produce inaccurate and inconsistent measurements, which may account for the unreliable results that have been found in a previous study. ${ }^{3}$ It has been suggested that neck flexion in the supine position will increase the atlanto-axial gap more than flexion in the erect or prone position. ${ }^{7}$ We found no significant difference between the erect and supine positions, and consider either would be acceptable for radiography. While lying supine against a head support can ensure full upper neck flexion, we prefer to use the erect position as this is generally more comfortable for the patient. However, a helper must be present to push the head back and keep the chin fully tucked in to touch the chest. Previously we had found that only one radiograph in flexion is necessary, as this produces the maximal atlanto-axial gap, ${ }^{4}$ which has been the case in other studies. ${ }^{89}$ A neutral view is best for showing abnormalities of the odontoid peg that may be associated with symptoms. ${ }^{6} 10 \mathrm{We}$ recommend an initial assessment with radiographs in neutral and flexion positions, and subsequent follow up radiographs in a flexion position only.

A recent study suggests that correction may be needed for magnification. ${ }^{8}$ We used the same radiography techniques throughout, so this factor would be constant in our study.

Over the course of five years in this group of children and young adults, there was an overall tendency for the atlanto-axial gap to decrease. This was statistically significant and consistent with the findings in our initial study in which there was a significant difference between the incidence of AAI in children aged 4-15, and a group of older individuals with Down's syndrome, of $10 \%$ and $2 \%$ respectively. ${ }^{4}$ This is presumably because the ligaments are excessively flexible in young people with Down's syndrome, and gradually become more firm with age. The most significant ligament in this context is the transverse ligament, binding the odontoid peg to the axis. There have been a few similar longitudinal studies of changes in the atlanto-axial gap over time in individuals with Down's syndrome. Ohsawa et al found an overall reduction in the atlanto-axial gap over five years in 69 individuals with Down's syndrome, but this was not statistically significant. ${ }^{11}$ Pueschel et al followed up 141 individuals over a maximum of 12 years and showed no particular changes ${ }^{12}$; this included nine individuals who initially had AAI, but lost it, and two who acquired AAI over the study period. Burke et al followed up 32 patients over 13 years including one who had AAI initially and lost it, and seven who were normal on the first study but acquired AAI later on. ${ }^{13}$ Our own study showed that only $13(19 \%)$ individuals increased the atlanto-axial gap over five years, and to a maximum of $2.0 \mathrm{~mm}$. None acquired AAI (defined as gap over $4.0 \mathrm{~mm}$ ) over the five years, who did not have this initially. Seven initially had AAI and this was maintained five years later in five of them. Our own study was of a complete and unselected cohort of all individuals with Down's syndrome attending school in our health district at the time of the first assessment. This, together with the use of the same radiography technique throughout, may account for the consistency of these results.

The only patient in our series with significant neck symptoms from AAI developed them acutely after ear, nose, and throat surgery. This was a boy who previously had a normal radiograph. It is recognised that acute AAI can occur during the course of anaesthesia, either from hyperextension during intubation, or due to excessive movement of the neck during anaesthesia. ${ }^{1415}$ Acute AAI with symptoms is also well recognised in Down's syndrome after any form of neck trauma, and is reported both in those with previously normal ${ }^{16}$ or abnormal radiographs. ${ }^{2}$ Our study underlines the fact that radiographs do not reliably screen for the likelihood of acute AAI after anaesthesia or trauma, and appropriate precautions for all individuals with Down's syndrome should be taken in these circumstances. For example, all individuals with Down's syndrome in our unit, undergoing anaesthesia, are fitted with a soft neck collar at premedication to alert all professionals about the risk of neck injury.

Our study identified a group of five individuals who had chronic AAI over the five year period, but who did not deteriorate radiologically or develop symptoms. In a review of all the published cases of symptomatic AAI in the literature, Pueschel et al found that over $80 \%$ developed symptoms gradually and did not suffer a particular traumatic episode. ${ }^{2}$ It is likely that those who gradually develop symptoms may be part of a small group with chronic AAI, as identified in our study. This raises the question of whether radiography can be used to identify individuals at increased risk of symptoms, and perhaps lead to intervention that will protect them.

Any programme of radiological screening for AAI must be with the awareness that symptoms from AAI are extremely rare. We had problems in only one out of 90 children with Down's syndrome over the course of five years. The usual incidence of symptoms is estimated at $1 \%$ to $2 \%$ of children ${ }^{2}$ and a radiograph can 
screen only for chronic AAI. Nevertheless, one could argue that symptoms when they occur can be serious, such as cord compression and paraplegia in individuals who may be poor at communicating what is wrong with them. There is evidence that the earlier surgery is performed when symptoms develop, the better the results. ${ }^{1718}$ The policy in our own health authority is to screen all children with Down's syndrome with a radiograph in the neutral and flexed position at the age of 4-5 years, before going into school, and maintaining particular vigilance for those found to have chronic AAI who receive repeat radiography at three to five yearly intervals in the flexed position only. Screening by radiography is relatively cheap and draws the attention of carers to neck problems in Down's syndrome, including the increased risk of acute neck injury. This radiological programme is backed up by regular checks for signs and symptoms of neck problems at a yearly medical check up.

What intervention should be provided for those found to have chronic AAI on radiography? The likely mechanism by which children with chronic AAI could increase the gap to the point when they may develop symptoms would be minor recurrent trauma causing hyperflexion of the neck. Over the course of our study, we restricted the sporting activities of those seven individuals who initially had chronic AAI, advising them against diving, trampolining, vigorous gymnastics, judo, and violent contact sports such as rugby, while encouraging such physical activities as running, jumping, swimming, and horseriding at slow speeds. None of these children deteriorated and developed symptoms. However, Cremers et al found that restricting sporting activities in 44 children with AAI over one year made no difference to the radiographs or clinical signs, compared with 47 with AAI who were not restricted. ${ }^{19}$ Similarly, Ohsawa et al reported on 14 patients with chronic AAI who remained symptom free over five years, despite lack of restriction on their sporting activities. ${ }^{11}$ The evidence, therefore, suggests restriction of activity is unnecessary, especially given the rarity of symptomatic AAI. For similar reasons, radiography cannot be justified before certain sporting activities that may cause excessive flexion of the neck, although carers should remain vigilant over the possible development of symptoms. This would apply particularly to those individuals found to have chronic AAI from radiological screening at a younger age.

We are very grateful to the $x$ ray departments at the Derbyshire Children's Hospital and Derby Royal Infirmary for their cooperation in this study, and also to Janet Floyd, secretary Ronnie MacKeith Centre, for typing the manuscript.

1 Locke GR, Gardner JI, Van Epps EF. Atlas-dens interval in children. AfR 1966; 97: 135-40.

2 Pueschel SM, Herndon JH, Gelch MM, Senft KE, Scola FH, Goldberg MJ. Symptomatic atlanto-axial subluxation in young persons with Down's syndrome. $¥$ Pediatr Orthop 1984; 4: 682-8.

3 Selby KA, Newton RW, Gupta S, Hunt L. Clinical predictors and radiological reliability in atlantoaxial subluxation in Down's syndrome. Arch Dis Child 1991; 66: 876-8.

4 Elliott S, Morton RE, Whitelaw RAJ. Atlantoaxial instability and abnormalities of the odontoid in Down's

5 McManners T. Odontoid hypoplasia. Br f Radiol 1983; 56: 907-10.
6 Eliott S. The odontoid process in children; is it hypoplastic? Clin Radiol 1988; 39: 391-3.

7 Prakash S. Radiological reliability in atlantoaxial subluxation [Letter]. Arch Dis Child 1992; 67: 256

8 Cremers MJG, Ramos L, Bol E, van Gijn J. Radiological assessment of the atlantoaxial distance in Down's assessment of the atlantoaxial distance

9 Jagjivan B, Spencer PAS, Hosking G. Radiological screening for atlanto-axial instability in Down's syndrome. Clin Radiol 1988; 39: 661-3.

10 Dawson EG, Smith L. Atlanto-axial subluxation in children due to vertebral anomalies. F Bone foint Surg Am 1979, 61: 582-7.

11 Ohsawa T, Izawa T, Kuroki Y, Ohmari K. Follow-up study of atlanto-axial instability in Down's syndrome withou separate odontoid process. Spine 1989; 14: 1149-53.

12 Pueschel SM, Scola FH, Pezzulo JC. A longitudinal study of atlanto-dens relationships in asymptomatic individuals with Down's syndrome. Pediatrics 1992; 89: 1194-8.

13 Burke SW, French HG, Roberts JM, et al. Chronic atlantoaxial instability in Down's syndrome. $\mathcal{F}$ Bone foint Surg Am 1985; 67: 1356-60

14 Msall ME, Reese RE, Di Gaudio K, Griswold K, Granger C, Cooke RE. Symptomatic atlanto-axial instability associated with medical and rehabilitative procedures in associated with medical and rehabilitative procedures in
children with Down's syndrome. Pediatrics 1990; 85: childre

15 Williams JP, Somerville GM, Miner ME, Reilly D. Atlantoaxial subluxation and trisomy 21 ; another peri-operative complication. Anaesthesiology 1987; 67: 253-4.

16 Davidson RG. Atlanto-axial instability in individuals with Down's syndrome; a fresh look at the evidence. Pediatrics 1988; 81: 857-65.

17 Martel W, Vyham R, Stimson CW. Subluxation of the atlas causing spinal cord compression in a case of Down's syndrome with a 'manifestation of occipital vertebra'. Radiology 1969; 93: 839-40.

18 Nordt JC, Stauffer ES. Sequelae of atlanto-axial stabilisation in 2 patients with Down's syndrome. Spine 1981; 6: 437-40.

19 Cremers MJG, Bol E, De Roos F, Van Gijn J. Risk of sports activities in children with Down's syndrome and atlantoaxial instability. Lancet 1993; 342: 511-4.

\section{Commentary}

Morton et al present data on a large series of patients and discuss an important and controversial management problem. The paper illustrates the many difficulties of using the measurement of the atlas-dens distance on the lateral cervical spine radiograph in the management of patients with Down's syndrome.

Subluxation and instability are, in my view often, wrongly, used interchangeably. The currently accepted definitions of AAI are an atlas-dens distance of $>4.0 \mathrm{~mm}^{1}$ or $>4.5 \mathrm{~mm}^{2}$ and, therefore, an absolute distance of $<4.0$ $\mathrm{mm}$ in children could be regarded as 'stable'. However, it is not as easy as this because stability (or instability) is a dynamic process and an atlas-dens distance $\geqslant 5.0 \mathrm{~mm}$ which remains unchanged between flexion and extension is not an unstable atlantoaxial joint whereas an atlas-dens distance which is, say, $1.0 \mathrm{~mm}$ in extension and $4.0 \mathrm{~mm}$ in flexion is unstable. Although the majority of published data indicate that the maximal gap occurs during flexion, use of this single view gives information only about subluxation - not instability.

Morton et al confirm the results of previous studies, ${ }^{34}$ which have shown that the atlasdens distance decreases with increasing age but they also confirm the finding that this change is as great, or less than, the change that can be measured on radiographs taken on the same occasion. ${ }^{5}$ Poor ossification of the odontoid peg, a slightly rotated film, or an uncooperative patient add to the difficulties of accurately measuring the atlas-dens distance. A reduction of AAI with increasing age is, as the authors suggest, probably due to the transverse ligament becoming firmer. However, a reduction 